\title{
Type of aortic valve replacement influences ascending aortic flow characteristics - a pilot study using 4D flow MRI
}

Florian von Knobelsdorff-Brenkenhoff ${ }^{*}$, Ralf F Trauzeddel ${ }^{1}$, Alex J Barker ${ }^{2}$, Henriette Gruettner ${ }^{1}$, Michael Markl ${ }^{2,3}$, Jeanette Schulz-Menger ${ }^{1}$

From 16th Annual SCMR Scientific Sessions

San Francisco, CA, USA. 31 January - 3 February 2013

\section{Background}

Prosthesis-related alterations of blood flow in the ascending aorta after aortic valve replacement (AVR) may influence aortic remodeling. The study aimed at analyzing ascending aortic flow characteristics after various types of AVR.

\section{Methods}

Flow-sensitive four-dimensional magnetic resonance imaging (4D-flow) was acquired in 38 AVR patients $(n=9$ mechanical, $n=8$ stentless bioprosthesis, $n=14$ stented bio- prosthesis, $\mathrm{n}=7$ autograft) and 9 healthy controls. Analysis included grading of vortex and helix flow (0-3 point scale), assessment of systolic flow eccentricity (1-3 point scale), and quantification of the segmental distribution of peak systolic wall shear stress (WSS peak $)$ in the ascending aorta.

\section{Results}

Compared to controls, mechanical prostheses showed the most distinct vorticity $(2.7 \pm 0.5$ vs. $0.7 \pm 0.7 ; \mathrm{p}<0.001)$, while stented bioprostheses exhibited most distinct helicity (2.6 \pm 0.7 vs. $1.6 \pm 0.5 ; \mathrm{p}=0.002)$ (Figures 1 and 2 ).

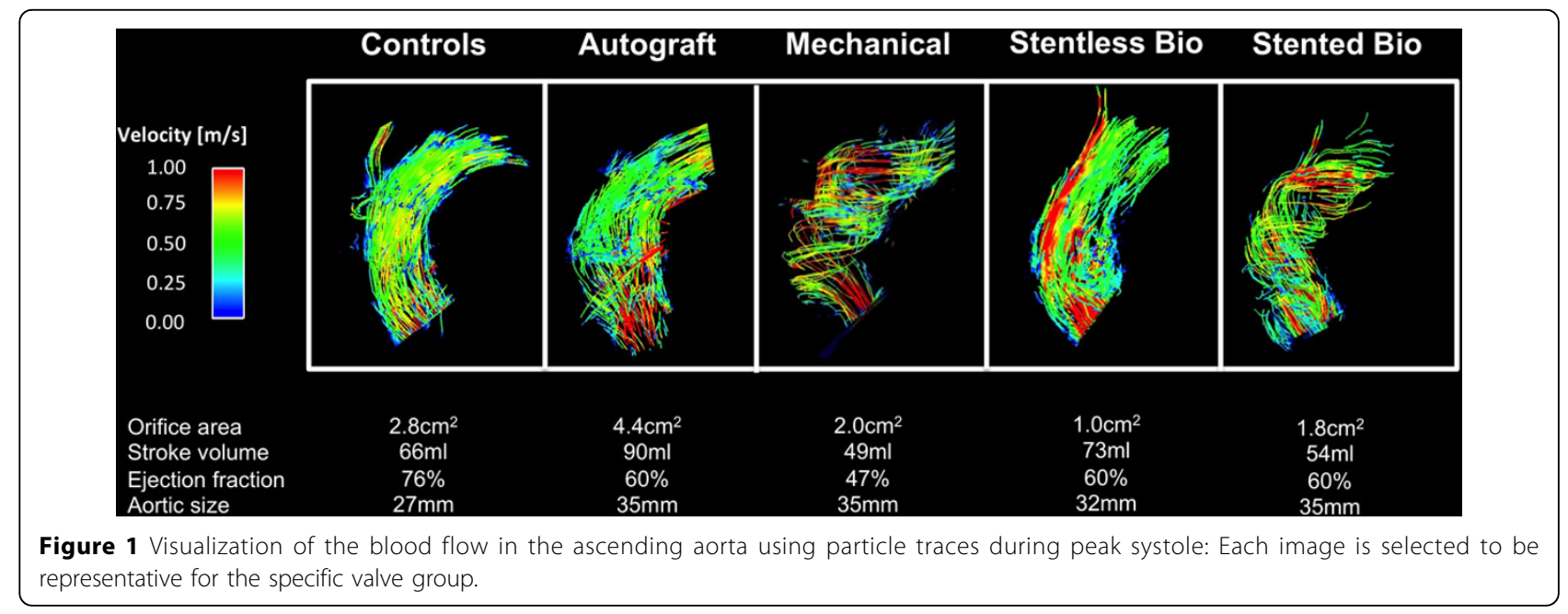

${ }^{1}$ Working Group Cardiovascular MRI, Experimental and Clinical Research Center (Charite Medical Faculty and MDC) and HELIOS Clinics Berlin, Berlin,

Germany

Full list of author information is available at the end of the article

(c) 2013 von Knobelsdorff-Brenkenhoff et al; licensee BioMed Central Ltd. This is an Open Access article distributed under the terms of 


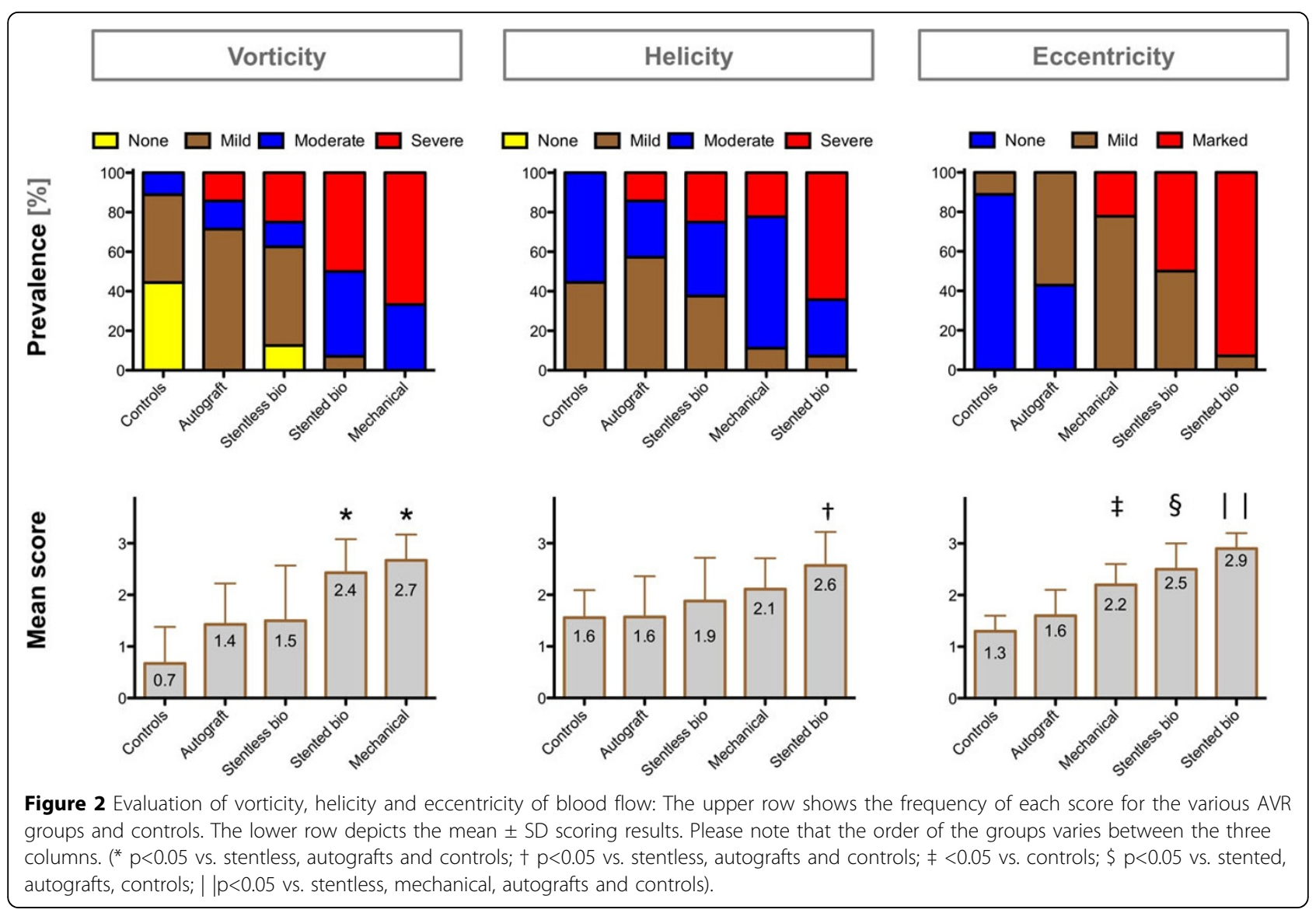

Instead of a physiologic central flow, all stented, stentless and mechanical prostheses showed eccentric flow jets mainly directed towards the right-anterior aortic wall. Stented and stentless prostheses showed an asymmetric distribution of $\mathrm{WSS}_{\text {peak }}$ along the aortic circumference, with significantly increased local $\mathrm{WSS}_{\text {peak }}$ where the flow jet impinged on the aortic wall. Local WSS peak was higher in stented $\left(1.4 \pm 0.7 \mathrm{~N} / \mathrm{m}^{2}\right)$ and stentless $(1.3$ $\left.\pm 0.7 \mathrm{~N} / \mathrm{m}^{2}\right)$ compared to autografts $\left(0.6 \pm 0.2 \mathrm{~N} / \mathrm{m}^{2}\right.$; $\mathrm{p}=0.005$ and $\mathrm{p}=0.008)$ and controls $\left(0.7 \pm 0.1 \mathrm{~N} / \mathrm{m}^{2}\right.$; $\mathrm{p}=0.017$ and $\mathrm{p}=0.027)$. Autografts exhibited lower absolute $\mathrm{WSS}_{\text {peak }}$ than controls $\left(0.4 \pm 0.1 \mathrm{~N} / \mathrm{m}^{2}\right.$ vs. $0.7 \pm 0.2 \mathrm{~N} /$ $\left.\mathrm{m}^{2} ; \mathrm{p}=0.003\right)$.

\section{Conclusions}

The flow characteristics in the ascending aorta in all AVR types were different from volunteers with native aortic valve, and they differed between the various types of AVR.

\section{Funding}

FvKB is supported by the Else Kröner-Fresenius Stiftung (Bad Homburg, Germany). AJB is funded by the Whitaker Postdoctoral and Fulbright Grants (New York,
USA). MM is supported by the NMH Excellence in Academic Medicine (EAM) Program 'Advanced Cardiovascular MRI Research Center’ (Chicago, USA).

\section{Author details}

${ }^{1}$ Working Group Cardiovascular MRI, Experimental and Clinical Research Center (Charite Medical Faculty and MDC) and HELIOS Clinics Berlin, Berlin, Germany. ${ }^{2}$ Department of Radiology, Feinberg School of Medicine, Northwestern University, Chicago, IL, USA. ${ }^{3}$ Department of Biomedical Engineering, McCormick School of Engineering, Northwestern University, Chicago, IL, USA

Published: 30 January 2013

doi:10.1186/1532-429X-15-S1-P245

Cite this article as: von Knobelsdorff-Brenkenhoff et al:: Type of aortic valve replacement influences ascending aortic flow characteristics - a pilot study using 4D flow MRI. Journal of Cardiovascular Magnetic Resonance 2013 15(Suppl 1):P245. 